\title{
Downregulation of high-affinity potassium and sodium symporter gene, EcHKT1;1, in Eucalyptus roots enhances salt tolerance
}

\author{
BALASUBRAMANIAN Aiyar ${ }^{1}$, Selvakesavan Rajendran kamalabai ${ }^{1}$, Shamili Krishnaraj ${ }^{1}$, \\ Sandhya M C ${ }^{1}$, Usha Jayachandran ${ }^{1}$, Sudha Selvam ${ }^{1}$, Siva kumar V1, Sowmiya \\ Kottaipalayam-Somasundaram ${ }^{1}$, Suryaprabha A C ${ }^{1}$, Vijaya Kumar Waman Bachpai ${ }^{1}$, \\ Hassen Gherbi ${ }^{2}$, Claudine Franche ${ }^{2}$, and Mathish Nambiar-Veetil ${ }^{1}$ \\ ${ }^{1}$ Institute of Forest Genetics and Tree Breeding \\ ${ }^{2}$ French National Research Institute for Sustainable Development
}

April 4, 2021

\begin{abstract}
Engineering for restricted root $\mathrm{Na}+$ uptake could potentially enhance salt tolerance in Eucalyptus. High-affinity K+ transporters (HKTs) have been implicated in Na+ uptake from the external medium as in the case of TaHKT2;1 or in the unloading of Na+ from xylem like in AtHKT1;1. To rapidly determine the in planta role of EcHKT1:1, composite transgenics in which EcHKT1:1 was specifically downregulated via RNAi in the roots were generated. Compared to the controls that failed to survive at 350 $\mathrm{mM} \mathrm{NaCl}, 33 \%$ of the composite transgenic plantlets generated using the EcHKT1;1 silencing construct were able to tolerate up to $400 \mathrm{mM} \mathrm{NaCl}$. In these composite transgenics, EcHKT1;1 downregulation ranged from $37 \%$ to $74 \%$. The average shoot to root ratio of sodium was 4.9 folds lower than the controls indicating restricted translocation of $\mathrm{Na}+$ to the shoots. Relative expression analysis in the leaves of two non-transgenic genotypes contrasting for their salt tolerance also showed downregulated EcHKT1;1 expression in the tolerant clone. The study thus determined that EcHKT1;1 is a major gene determining Na+ transport from the roots to shoots. This study also demonstrated the utility of the composite transgenic approach for screening genes conferring salt tolerance in tree species.
\end{abstract}

\section{Hosted file}

Main document PCE-EcHKT1_1.pdf available at https://authorea.com/users/405678/articles/ 516575-downregulation-of-high-affinity-potassium-and-sodium-symporter-gene-echkt1-1-ineucalyptus-roots-enhances-salt-tolerance 

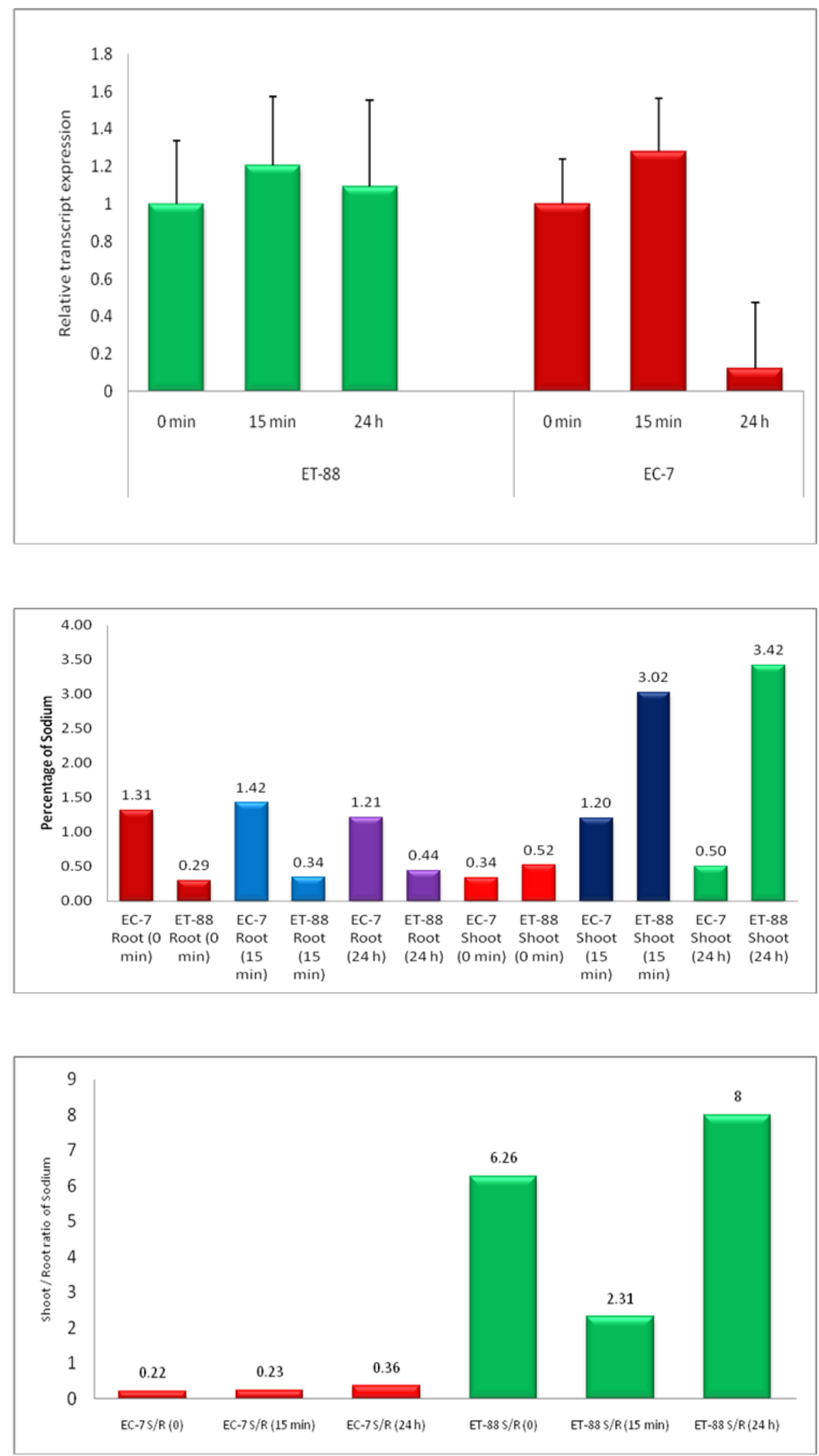

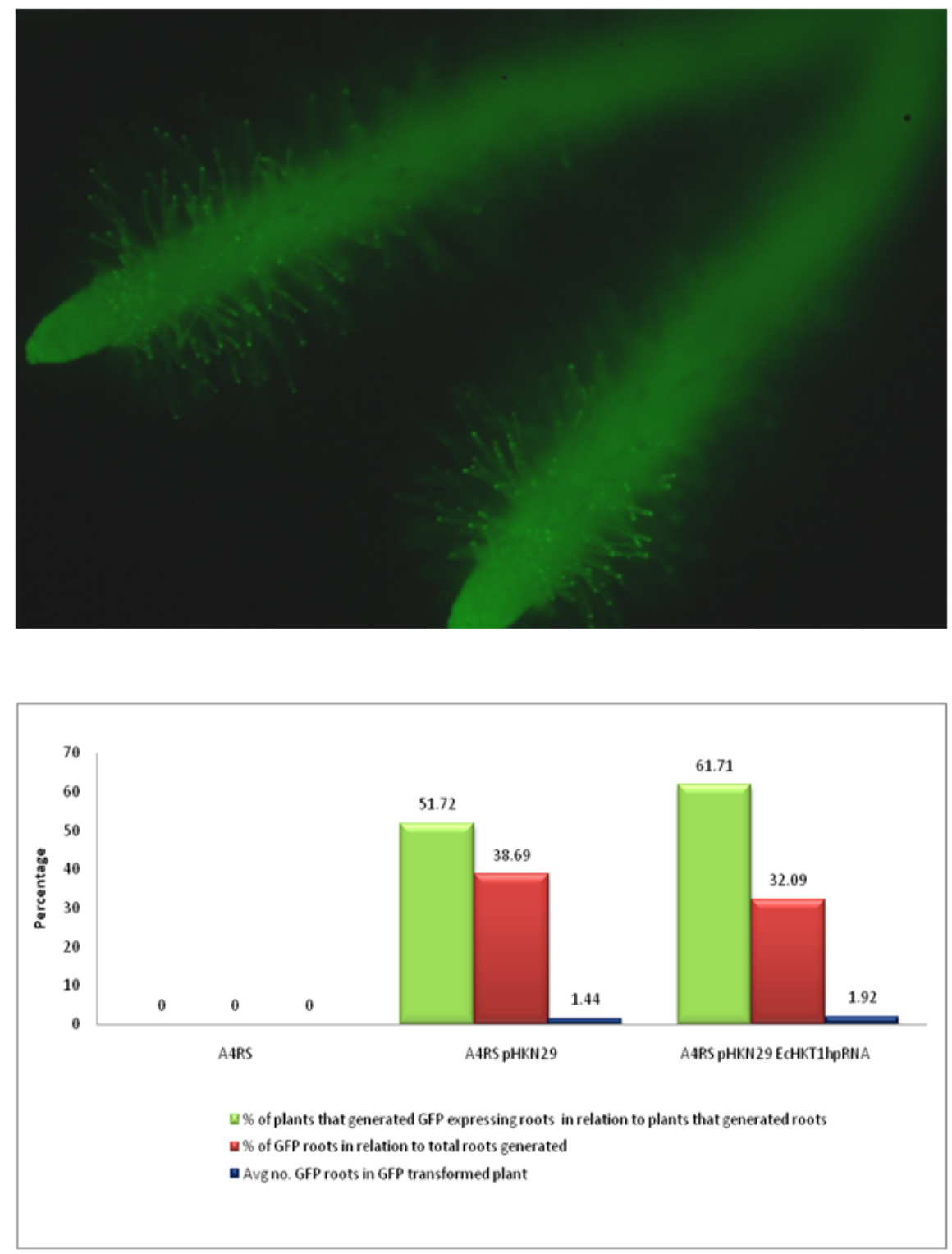

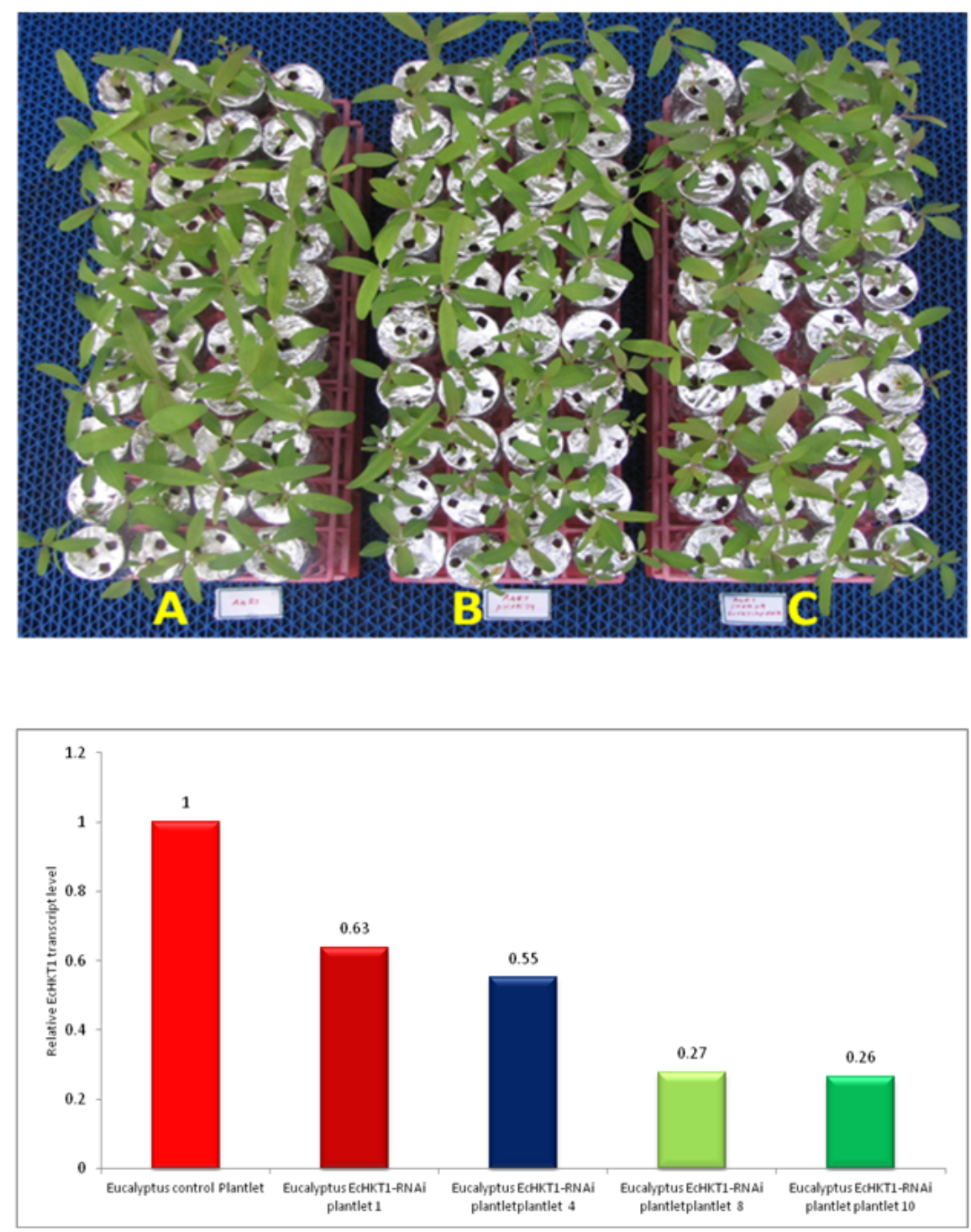

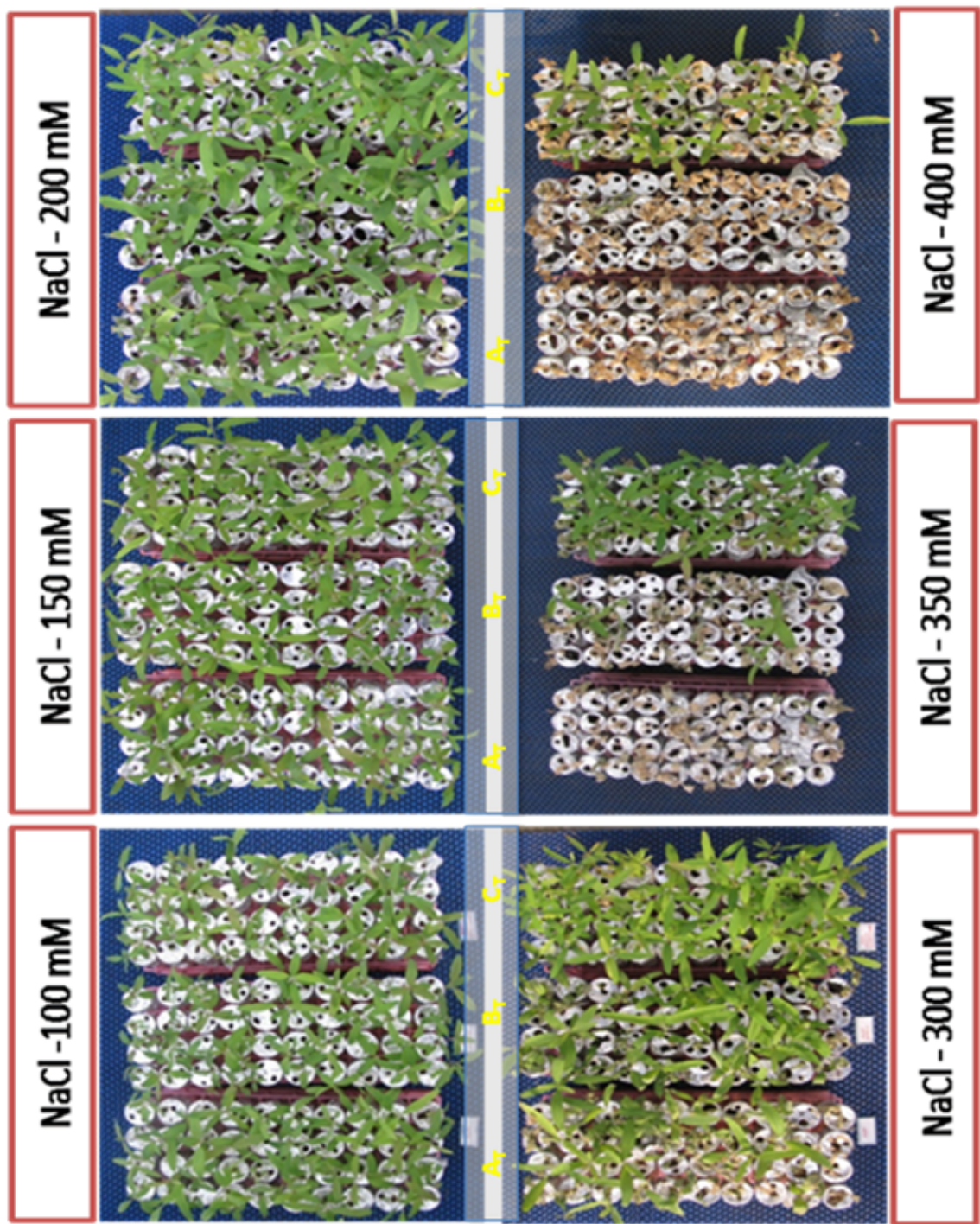

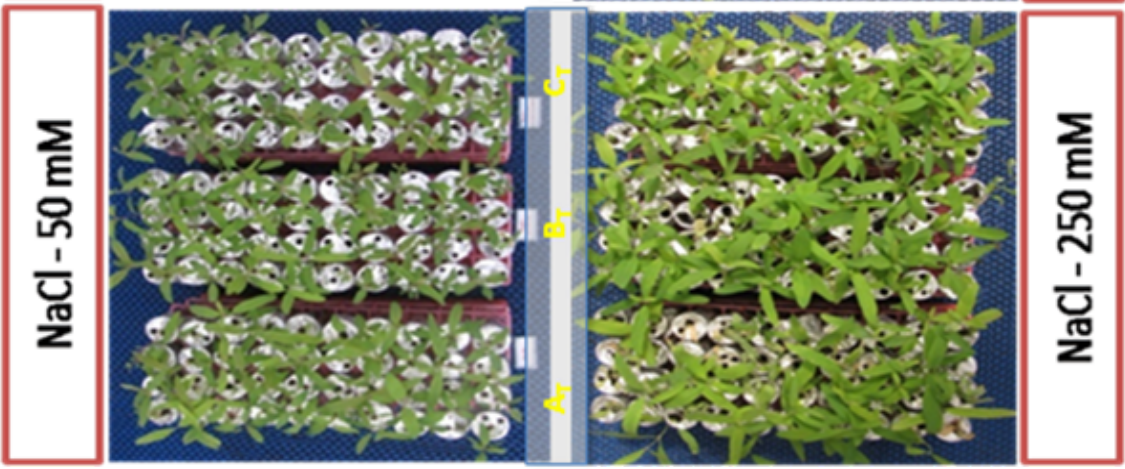



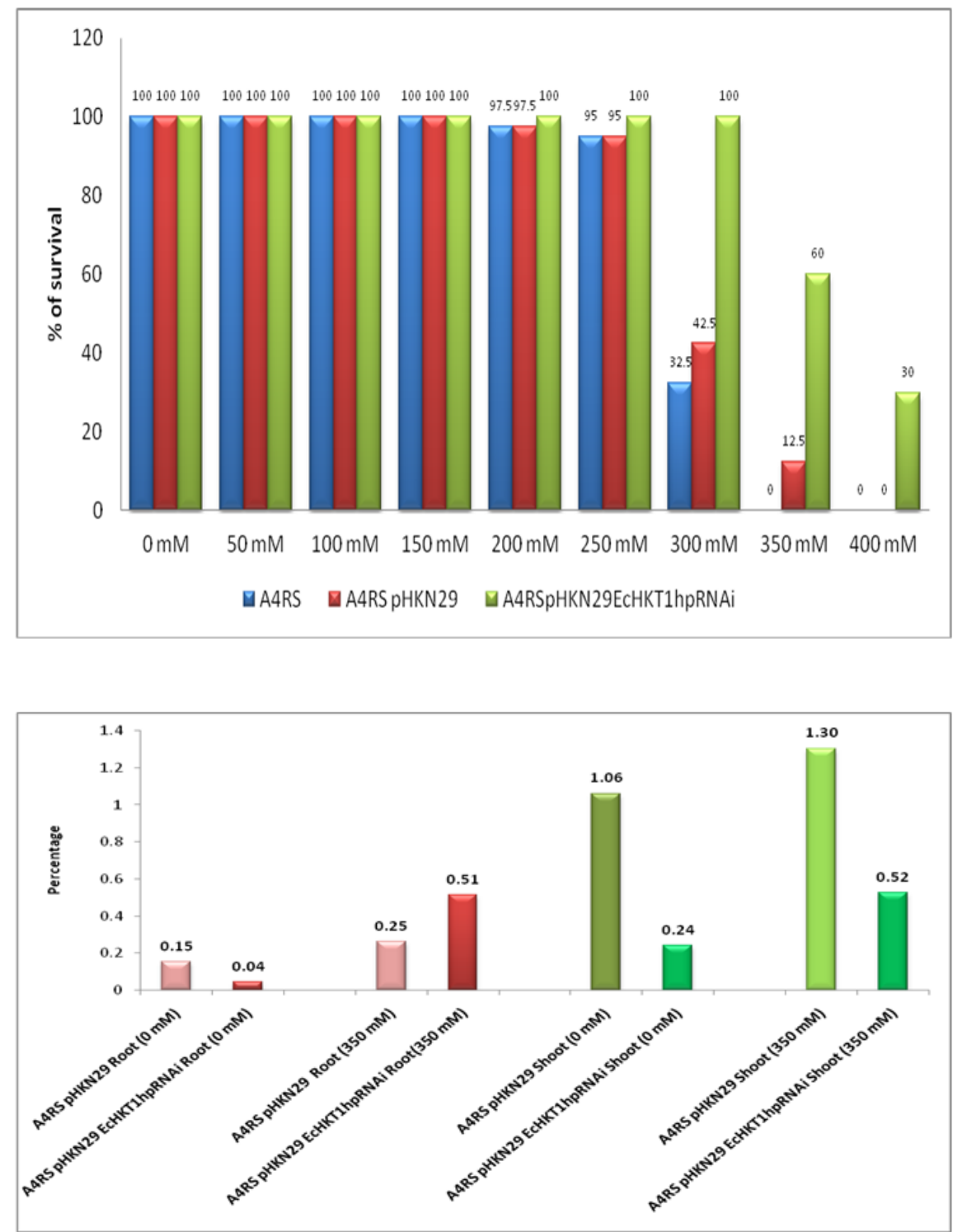


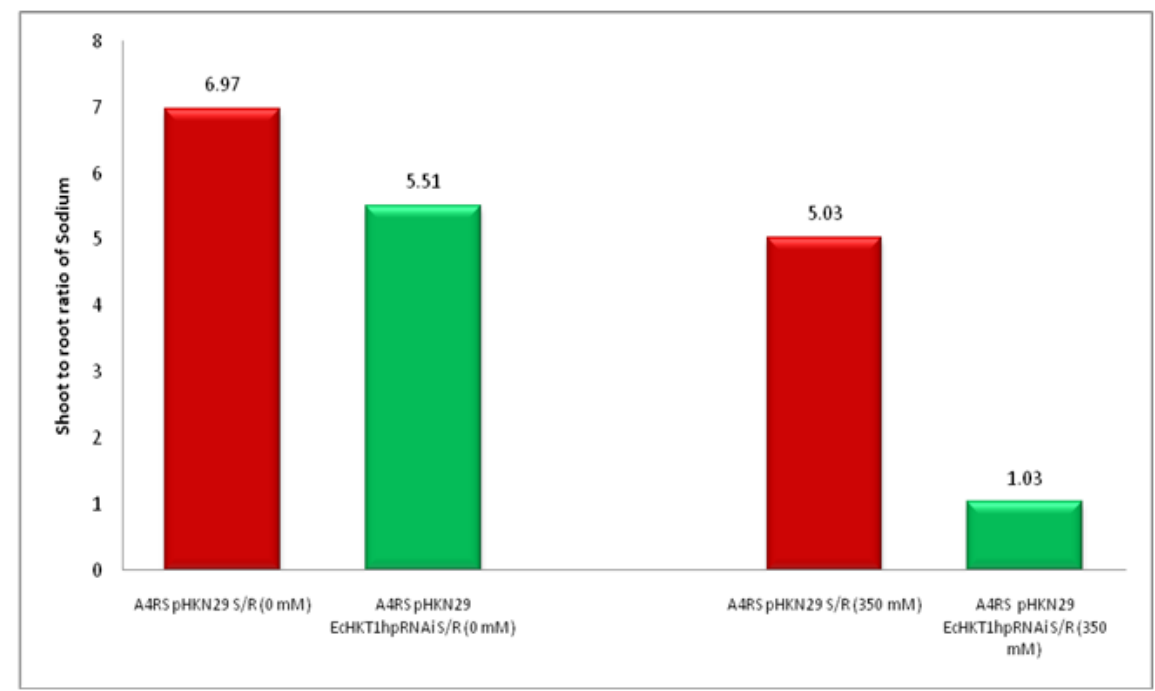

